\title{
Quantitative evaluation of CpG island methylation in hyperplastic polyps
}

\author{
Cecily P Vaughn ${ }^{1}$, Andrew R Wilson ${ }^{1}$ and Wade S Samowitz ${ }^{1,2}$ \\ ${ }^{1}$ ARUP Institute for Clinical and Experimental Pathology, Salt Lake City, UT, USA and ${ }^{2}$ Department of \\ Pathology, University of Utah Health Sciences Center, Salt Lake City, UT, USA
}

\begin{abstract}
Microsatellite unstable cancers account for up to $15 \%$ of sporadic colon cancers and are predominantly located in the proximal colon. These cancers commonly show MLH1 promoter methylation and the CpG island methylator phenotype (CIMP). A potential precursor of sporadic unstable cancers, the proximal hyperplastic polyp, is also reported to have CIMP and MLH1 methylation. However, this latter finding is not supported by MLH1 protein expression studies. To help resolve this apparent discrepancy, we determined MLH1 promoter methylation and CIMP by quantitative real-time PCR for 29 proximal hyperplastic polyps, 23 distal hyperplastic polyps, and 11 sporadic microsatellite unstable colon cancers. BRAF V600E mutation status was also determined. Positive methylation was defined as the percentage of methylated reference (PMR) $>10$. Only 1 of 29 proximal hyperplastic polyps showed positive MLH1 methylation (PMR of 13.0). Neither this polyp nor seven other proximal polyps with PMR values between 0 and 10 showed loss of MLH1 protein expression by immunohistochemistry. In contrast, all 11 microsatellite unstable cancers showed high degrees of MLH1 methylation, with PMR values $>30$. Fourteen of twenty-nine $(48 \%)$ of the proximal hyperplastic polyps and 1 of $23(4 \%)$ of the distal hyperplastic polyps showed CIMP $(P<0.001)$. Of the unstable cancers, 10 of 11 showed CIMP. The PMR values in the CIMP-positive proximal hyperplastic polyps were significantly lower than those of the unstable cancers for 4 of the 5 CIMP markers $(P<0.05)$. BRAF V600E mutations were seen in $83 \%$ of proximal and $74 \%$ of distal hyperplastic polyps. Quantitative analysis of $M L H 1$ methylation does not support earlier reports of MLH1 methylation in proximal hyperplastic polyps. However, these lesions do harbor promoter methylation at other CIMP loci, although at a lower level than that seen in unstable cancers. If these polyps are the precursor for sporadic microsatellite unstable cancers, then MLH1 methylation and higher degrees of promoter methylation in general occur at a later stage of carcinogenesis.
\end{abstract}

Modern Pathology (2010) 23, 151-156; doi:10.1038/modpathol.2009.150; published online 23 October 2009

Keywords: $\mathrm{CpG}$ island methylator phenotype; hyperplastic polyp; $\mathrm{MLH1}$; promoter methylation

Although most colon cancers are thought to arise through the adenoma-carcinoma pathway, an alternative 'serrated pathway' is now well recognized. ${ }^{1}$ Cancers arising through this pathway are characterized by microsatellite instability (MSI), rather than the chromosomal instability seen in the adenomacarcinoma pathway. ${ }^{1,2}$ Microsatellite unstable cancers account for up to $15 \%$ of sporadic colon cancers and are predominantly located in the proximal colon. $^{3}$ In addition to MSI, these cancers also commonly exhibit widespread CpG island methyla-

Correspondence: Dr WS Samowitz, MD, Department of Pathology, University of Utah Health Sciences Center, ARUP Laboratories, 500 Chipeta Way, Salt Lake City, UT 84108, USA.

E-mail: wade.samowitz@aruplab.com

Received 26 May 2009; revised and accepted 11 August 2009; published online 23 October 2009 tion, or the $\mathrm{CpG}$ island methylator phenotype (CIMP), and BRAF mutations. ${ }^{4,5}$ In sporadic cancers, MSI typically arises from the loss of MLH1 expression due to promoter methylation. ${ }^{6}$

Traditionally hyperplastic polyps were presumed to be non-neoplastic without malignant potential; however, these lesions, or at least a subset of these lesions, are now presumed to be the precursor in the 'serrated pathway'., ${ }^{1,2}$ Thus, multiple studies have been undertaken to better characterize hyperplastic polyps and to determine which are clinically relevant. $^{2,7-11}$ Like microsatellite unstable cancers, these polyps have been shown to exhibit CIMP $^{7-11}$ and BRAF mutations; ${ }^{7-11}$ however, they do not show high level microsatellite instability. ${ }^{7-10}$ Hyperplastic polyps have also been reported to have MLH1 promoter methylation. ${ }^{7-9}$ This finding, however, is somewhat puzzling given that these lesions do not 
show loss of MLH1 protein expression by immunohistochemistry. ${ }^{7,12}$

Assessments of promoter methylation in MLH1 and other genes have typically been performed using methylation-specific PCR with agarose gelbased detection. Recently, quantitative methods, including MethyLight, have been employed to study methylation status in multiple cancers. ${ }^{13-16}$ MethyLight employs both methylation-specific primers for amplification and methylation-specific probes for detection. In addition, amplification of a reference gene allows for normalization of the input DNA and provides a semi-quantitative assessment of the degree of methylation.

Although MethyLight has been employed for the assessment of methylation in microsatellite unstable colon cancers, ${ }^{5,17}$ it has not been used in the characterization of potential precursor lesions. As the MLH1 methylation reported in previous studies of hyperplastic polyps does not correspond to loss of MLH1 protein expression or MSI, we examined promoter methylation levels in these lesions using MethyLight to determine if quantitative measurement might provide a more biologically relevant assessment. In addition, as sporadic microsatellite unstable cancers are predominantly located in the proximal colon, and if hyperplastic polyps are indeed a precursor to these cancers, then a comparison of the molecular characteristics of proximal and distal polyps is of particular interest. To this end, we evaluated promoter methylation of $M L H 1$ and other CIMP markers in proximal and distal hyperplastic polyps. For comparison we also examined methylation status in microsatellite stable and unstable cancers, and normal mucosa. Results of MLH1 methylation were compared with MLH1 protein immunostaining for corresponding samples. In addition, we examined the frequency of BRAF mutations in these lesions.

\section{Materials and methods}

\section{Specimens}

A search of hyperplastic polyps from the dates August 2007 to January 2008 was performed from the University of Utah surgical pathology files. At this time the term 'hyperplastic polyp' was used to refer to any non-dysplastic serrated lesion, including lesions that have otherwise been termed 'sessile serrated adenomas' or 'serrated polyps with abnormal proliferation. ${ }^{\prime 8,18}$ We did not include any mixed lesions or cases with hyperplastic polyposis. ${ }^{19}$ The specimens consisted of 29 proximally located hyperplastic polyps, from 29 patients, and 23 distally located (splenic flexure, descending colon and rectum) hyperplastic polyps, from 23 patients. Polyp sizes obtained from endoscopy reports showed that 10 of 26 proximal and 4 of 23 distal polyps were $0.5 \mathrm{~cm}$ or larger. (Sizes were not available for three of the proximal hyperplastic polyps.).

In addition, 11 microsatellite unstable cancers, 19 microsatellite stable cancers, and 12 proximal normal mucosa specimens were also included. Of the 19 microsatellite stable cancers, 11 were proximally located and eight were distally located. The normal mucosa specimens were obtained from 12 of the cases with proximal hyperplastic polyps. All of the microsatellite unstable cancers had previously been determined to be positive for the BRAF V600E mutation and to lack MLH1 protein expression. This was done in an attempt to avoid unstable cancers associated with hereditary non-polyposis colorectal cancer (HNPCC or Lynch syndrome).

All samples were obtained with approval from the University of Utah IRB.

\section{DNA Extraction}

Microdissection and DNA extraction was performed as described previously. ${ }^{20}$ Areas of interest within the biopsies were identified using hematoxylin- and eosin-stained sections. Tissue was then microdissected from corresponding aniline blue-stained slides. Dissected tissue was treated with $200 \mu \mathrm{g}$ of Proteinase K (Qiagen, Valencia, CA, USA) in $200 \mu \mathrm{l}$ TE buffer with $1 \%$ Tween 20 overnight at $65^{\circ} \mathrm{C}$. Samples were then boiled for $10 \mathrm{~min}$ and centrifuged. No additional purification of the DNA was performed.

\section{Promoter Methylation Assessment by MethyLight}

The methylation status of the promoter regions of MLH1, CACNA1G, IGF2, NEUROG1, RUNX3, and SOCS1 were determined using MethyLight as previously described. ${ }^{13-15}$ DNA lysates were first sodium bisulfite treated using the EZ DNA Methylation Kit (Zymo Research, Orange, CA, USA). All steps were performed according to the kit protocol.

Converted DNA was amplified by real-time PCR using previously described ${ }^{5}$ methylation-specific primers. The DNA was also amplified for COL $2 A 1^{16}$ to allow for normalization of the amount of input converted DNA. Methylation-specific dual-labeled probes were included in each reaction. Primer and probe sequences are available in Supplementary Table 1. Each $20 \mu \mathrm{l}$ reaction contained $2 \mu \mathrm{l}$ of bisulfite-treated DNA, $0.3 \mu \mathrm{M}$ each primer, $0.1 \mu \mathrm{M}$ probe, and $1 \times$ AmpliTaq Gold PCR Master Mix (Applied Biosystems, Foster City, CA, USA). Amplification was performed on the Applied Biosystems $7900 \mathrm{HT}$ and entailed an initial denaturation at $95^{\circ} \mathrm{C}$ for $2 \mathrm{~min}$ followed by 45 cycles of denaturation $\left(95^{\circ} \mathrm{C}\right.$ for $\left.10 \mathrm{~s}\right)$ and annealing/elongation $\left(60^{\circ} \mathrm{C}\right.$ for $1 \mathrm{~min})$.

Standard curves for each target reaction were generated by amplifying serial dilutions of sodium bisulfate-converted Universal Methylated Human 
DNA Standard (Zymo Research). The level of methylation (percentage of methylated reference (PMR)) in each DNA sample was determined by comparing the crossing threshold of each reaction to its respective standard curve and then calculating the ratio of the concentration of the target gene to the concentration of COL2A1 and multiplying by 100 .

\section{The CpG Island Methylator Phenotype}

CpG island methylator phenotype (CIMP) status was determined using the criteria set forth by Weisenberger. ${ }^{5}$ This CIMP panel consists of CpG islands from the following genes: CACNA1G, IGF2, NEUROG1, RUNX3, and SOCS1. PMR values $>10$ were defined as positive, and the designation of CIMP was applied to all samples with methylation at 3 or more loci. As MLH1 is not part of this panel, methylation of the promoter region of this gene was considered separately.

In addition to the determination of CIMP status, PMR values of the CIMP-positive proximal hyperplastic polyps and the CIMP-positive sporadic microsatellite unstable cancers were compared.

\section{MLH1 Immunohistochemistry}

All hyperplastic polyp samples that showed any level of MLH1 promoter methylation (even PMRs between 0 and 10) were subsequently analyzed for MLH1 protein expression. All staining steps were performed on the automated immunostainer from Ventana Medical Systems (Tucson, AZ, USA). A mouse monoclonal antibody for MLH1 (clone G16815; BD Pharmingen, San Diego, CA, USA) was applied for $32 \mathrm{~min}$ at a dilution of 1:40. The IView DAB detection kit (Ventana Medical Systems) was used for detection, and sections were counterstained with hematoxylin. ${ }^{21}$

\section{BRAF V600E Assessment}

The DNA samples were analyzed for the BRAF V600E mutation by melting curve analysis on the LightCycler (Roche Applied Science, Indianapolis, IN, USA). Samples were amplified for $B R A F$ exon 15 using the forward primer $5^{\prime}$-CTCTTCATAATGCCT GCTCTGATAGG-3' ${ }^{\prime}$ and the reverse primer $5^{\prime}$-TAGT AACTCAGCAGCATCTCAGG $-3^{\prime}$. The reaction also included the following hybridization probes: $5^{\prime}$-AG CTACAGTGAAATCTCGATGGAG-fluorescein- $3^{\prime}$ and 5'-LCRed 640-GGTCCCATCAGTTTGAACAGTTGTC TGGA- $3^{\prime}$. Each $20 \mu \mathrm{l}$ reaction contained $0.2 \mu \mathrm{l}$ DNA lysate, $0.5 \mu \mathrm{M}$ each primer, $0.2 \mu \mathrm{M}$ each probe, $1 \times$ Roche LightCycler DNA Master HybProbe (containing Taq polymerase, dNTPs, and buffer), and $3 \mathrm{mM}$ $\mathrm{MgCl}_{2}$. Cycling entailed initial denaturation at $95^{\circ} \mathrm{C}$ for $5 \mathrm{~min}$ followed by 45 cycles of denaturation $\left(94^{\circ} \mathrm{C}\right.$ for $\left.1 \mathrm{~s}\right)$, annealing $\left(55^{\circ} \mathrm{C}\right.$ for $\left.20 \mathrm{~s}\right)$, and elongation $\left(72^{\circ} \mathrm{C}\right.$ for $\left.40 \mathrm{~s}\right)$. Amplification was followed by a melting from 45 to $72^{\circ} \mathrm{C}$ at a rate of $0.1^{\circ} \mathrm{C} /$ second.

Melting curve analysis was performed using the LightCycler software, version 3.5. Melting curves with a $\mathrm{T}_{M}$ of $64-66^{\circ} \mathrm{C}$ are indicative of a wild-type sequence. Melting curves with a $\mathrm{T}_{\mathrm{M}}$ of $58-60^{\circ} \mathrm{C}$ are indicative of a V600E mutant sequence.

\section{Statistical Analysis}

Two-sample proportion tests were used to compare the differences in CIMP-positive frequency between the proximal hyperplastic polyps and the other sample types. The Wilcoxon two-sample test was used to compare the PMR values of the CIMPpositive hyperplastic polyps and the PMR values of the CIMP-positive sporadic microsatellite unstable colon cancer samples at each locus. Results were considered statistically significant for $P<0.05$. All calculations were performed using SAS software, version 9.1 of the SAS System Copyright 2002-2003 SAS Institute, Cary, NC, USA.

\section{Results}

\section{MLH1 Methylation}

Only 1 of 29 of the proximal hyperplastic polyp samples showed MLH1 promoter methylation, with a PMR of 13.0. An additional seven proximal hyperplastic polyps showed very low levels of methylation with PMR values $<10$. None of the distal hyperplastic polyps showed MLH1 promoter methylation. In contrast, all of the sporadic microsatellite unstable cancers showed MLH1 promoter methylation, with PMR values all greater than 30 . The 19 microsatellite stable cancers and 12 proximal normal mucosa samples were negative for any level of MLH1 promoter methylation.

\section{MLH1 Immunohistochemistry}

Immunohistochemistry results for the eight proximally located hyperplastic polyps that showed any level of MLH1 methylation, including the polyp with PMR of 13.0, showed normal MLH1 protein expression.

\section{CpG Island Methylator Phenotype}

Fourteen of 29 (48\%) proximal hyperplastic polyps showed CIMP (three or more methylated CpG islands with PMR >10, excluding MLH1), whereas only 1 of 23 (4\%) distal hyperplastic polyps showed CIMP. This difference was statistically significant $(P<0.001)$, as shown in Table 1 . In comparison, 10 of 11 of the sporadic microsatellite unstable cancers 
Table 1 Comparison of CIMP-positive proportions

\begin{tabular}{lcccc}
\hline Sample type & Total & $\begin{array}{c}\text { CIMP- } \\
\text { positive }\end{array}$ & Proportion & P-value \\
\hline $\begin{array}{l}\text { Proximal hyperplastic } \\
\text { polyps }\end{array}$ & 29 & 14 & $48 \%$ & $* *$ \\
$\begin{array}{l}\text { Distal hyperplastic } \\
\text { polyps }\end{array}$ & 23 & 1 & $4 \%$ & $<0.001$ \\
Unstable cancers & 11 & 10 & $91 \%$ & 0.014 \\
$\begin{array}{l}\text { Stable cancers, proximal } \\
\text { Stable cancers, distal }\end{array}$ & 11 & 0 & $0 \%$ & 0.004 \\
Normal mucosa & 12 & 0 & $0 \%$ & 0.013 \\
\hline
\end{tabular}

${ }^{\mathrm{a}}$ Proportions test against proximal hyperplastic polyps.

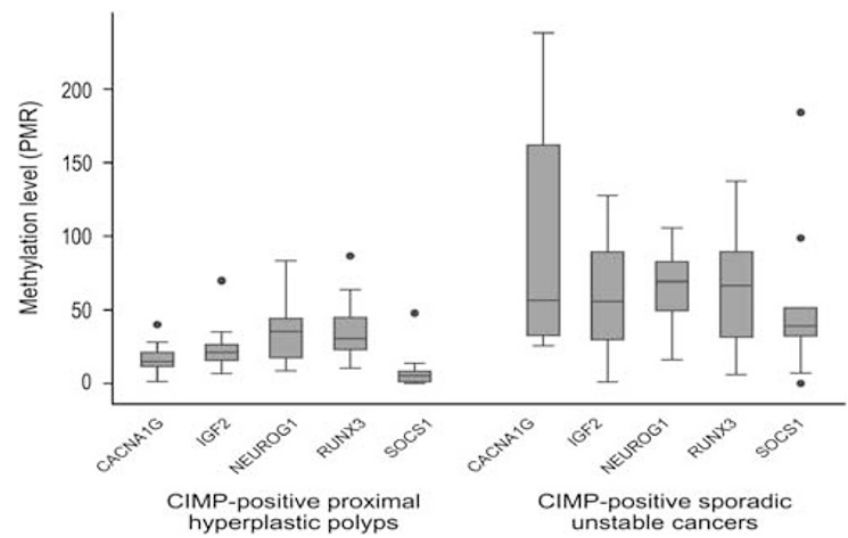

Figure 1 Relative distribution of methylation levels at individual loci in CIMP-positive samples. The methylation levels (percentage of methylated reference, or PMR) were statistically significantly different between proximal hyperplastic polyps and microsatellite unstable cancers for all genes except RUNX3 (Wilcoxon pairwise comparison $P$-values $<0.05$ ).

were positive for CIMP. None of the stable cancers or normal mucosa samples showed CIMP.

CIMP was seen in 7 of 16 proximal hyperplastic polyps $<0.5 \mathrm{~cm}$ and 6 of 10 proximal hyperplastic polyps $0.5 \mathrm{~cm}$ or larger. The single distal hyperplastic polyp with CIMP was smaller than $0.5 \mathrm{~cm}$.

\section{Quantitative Comparison of Methylation Levels}

A comparison of the methylation levels in CIMPpositive proximal hyperplastic polyps and CIMPpositive microsatellite unstable cancers is shown in Figure 1. (CIMP-negative samples were not included so as to provide a direct comparison of samples with widespread methylation). The PMR values in the proximal hyperplastic polyps were lower than the corresponding PMR values in the unstable cancers for all five $\mathrm{CpG}$ islands; this was statistically significant in four of the five $\mathrm{CpG}$ islands.

\section{BRAF Mutation Status}

The BRAF V600E mutation was detected in 24 of 29 $(83 \%)$ of the proximally located hyperplastic polyps. This included 13 of 14 CIMP-positive and 11 of 15 CIMP-negative proximal polyps. In the distal hyperplastic polyps, 17 of $23(74 \%)$ harbored the BRAF V600E mutation, including the single CIMP-positive sample. Of the 18 stable cancers that were successfully amplified, only two harbored the BRAF V600E mutation. Both of these stable cancers were proximally located; however, neither of them showed CIMP.

\section{Discussion}

A semi-quantitative assessment of $M L H 1$ promoter methylation does not support previous reports of MLH1 methylation in hyperplastic polyps of either the proximal or distal colon. Previous studies have used methylation-specific PCR and gel-based detection. ${ }^{2,7-9}$ As originally described, this method can detect methylated alleles present at a prevalence of $0.1 \%{ }^{22}$ Although we did see evidence of some methylation in 8 of 29 proximal hyperplastic polyps, only one of these met the minimum requirement of a PMR of 10, and the PMR for this polyp was only 13. In contrast, PMR values greater than 30 were observed in all of the sporadic microsatellite unstable cancers. Subsequent immunohistochemical staining for MLH1 in the eight hyperplastic polyp samples in this study with any degree of MLH1 methylation showed the presence of MLH1 protein. Thus, previous reports of MLH1 methylation in polyps that showed no loss of MLH1 protein or associated microsatellite instability may be explained by the oversensitivity of methylationspecific PCR. The apparent contradiction between the detection of $M L H 1$ promoter methylation and the preservation of MLH1 protein expression has been noted before. ${ }^{7}$ It was suggested that this might be because of the detection of hemimethylation of alleles which would not result in complete loss of expression. Our current results, in which a semi-quantitative assay shows little if any MLH1 methylation, suggest that the previously used nonquantitative methylation-specific PCR may have been overly sensitive and that significant, biologically relevant MLH1 methylation was simply not present.

Although we did not observe MLH1 methylation, CIMP was observed in approximately half of the proximally located hyperplastic polyp samples in this study. There is a significant difference between these polyps and those located in the distal colon, where only 1 of 23 were CIMP-positive. Differences between methylation levels in these two locations have previously been reported, ${ }^{8}$ although here the use of a semi-quantitative assessment further emphasizes this distinction. The proportion of CIMP in proximally located polyps is also significantly greater than that seen in proximal and distal stable cancers and in normal mucosa samples. The presence of CIMP in proximal hyperplastic polyps 
supports their role as a precursor to the sporadic unstable cancers, of which 10 of 11 were CIMPpositive. However, even among CIMP-positive samples, there is a distinction between the methylation seen in proximally located hyperplastic polyps and unstable cancers. A comparison between the PMR values for the CIMP-positive proximal polyps and the microsatellite unstable cancers showed significantly higher methylation in the unstable cancers (see Figure 1).

It should be noted that the frequency of CIMP and MLH1 methylation in unstable cancers seen in this study (91 and 100\%, respectively) is slightly higher than that seen in some previous studies; for example, Ogino et $a l^{23}$ reported CIMP in $73 \%$ of unstable cancers and in our previous study ${ }^{4}$ we reported MLH1 methylation in $72.5 \%$ of these tumors. This is probably because the unstable cancers evaluated in this study were chosen based upon the presence of $B R A F$ mutations and loss of MLH1 immunostaining. This was done to exclude, as much as possible, microsatellite unstable cancers associated with Lynch syndrome (HNPCC), as these tumors typically do not harbor $B R A F$ mutations. ${ }^{24}$ The precursor of Lynch syndromeassociated colorectal adenocarcinomas is considered to be the adenomatous polyp; ${ }^{25,26}$ because we were trying to study the relevance of hyperplastic polyps to sporadic unstable cancers, Lynchassociated tumors would not have been an appropriate reference point.

The BRAF V600E mutation, in contrast to MLH1 methylation and CIMP, appears to be a common and early event in proximally located hyperplastic polyps. Similar results have been observed in numerous studies. ${ }^{7,9-11}$ However, there seems to be no appreciable difference between the frequency of BRAF mutation seen in proximal hyperplastic polyps and distal hyperplastic polyps. The high level of BRAF mutations in both proximal and distal hyperplastic polyps suggests that although this may be an early event in the development of sporadic unstable cancers, it is not a useful marker for identifying clinically relevant serrated pathway precursors. In addition, and in contrast to the situation with colorectal cancers, ${ }^{4,5}$ no correlation between CIMP and BRAF mutations in hyperplastic polyps was observed (data not shown).

In this study, we chose not to histologically classify types of hyperplastic polyps, but rather to focus on their location within the colon. Polyp site is important because microsatellite unstable cancers predominantly occur in the proximal colon, and thus one would expect the associated precursor lesions to also present there. We then used a strictly molecular approach to try to differentiate polyps that may be biologically relevant precursors from those which are not. Previously used histological designations, which have centered on fairly subtle architectural criteria, can be difficult to reproduce. $^{27,28}$ Moreover, in our experience the archi- tectural features deemed to be important are seen in most proximally located hyperplastic polyps. Regardless, when defined histologically, sessile serrated polyps, the putative precursor lesion, appear to comprise $\sim 40 \%$ of the proximally located hyperplastic polyps ${ }^{8,18}$ and thus should have been amply represented in our study.

The semi-quantitative nature of MethyLight provides additional insight into the nature of methylation in hyperplastic polyps. This method is able to differentiate between high levels of methylation and low levels that may not be biologically relevant. The quantitative measurement performed here identified, at most, only low levels of $M L H 1$ methylation in hyperplastic polyps and thus provides an explanation for the previously discordant observation that MLH1 expression, as measured by immunostaining, is preserved in these lesions. This semi-quantitative assessment also showed that while approximately half of proximal hyperplastic polyps exhibit CIMP, the degree of methylation at individual loci is less than that seen in sporadic microsatellite unstable cancers. In addition, we observed that the frequency of CIMP in proximal hyperplastic polyps was much higher than that in distal hyperplastic polyps, suggesting that proximally located hyperplastic polyps may be precursors in the progression to unstable tumors and, perhaps, to CIMP high stable cancers, both of which are also predominantly located in the proximal colon. BRAF mutations, however, do not appear to be localized in the proximal colon. Lastly, if hyperplastic polyps are precursor lesions, then MLHI methylation and high levels of CIMP occur at a later stage of carcinogenesis. These results are consistent with studies of hyperplastic polyps in continuity with dysplasia and/or cancer, as loss of MLH1 immunostaining only occurs in the setting of dysplasia or cancer. ${ }^{12}$

In summary, this study, which represents the first quantitative assessment of promoter methylation in hyperplastic polyps, provides an explanation for previous contradictory results with respect to $M L H 1$ methylation, MLH1 immunohistochemistry, and MSI in these lesions. If these polyps are the precursor for sporadic microsatellite unstable cancers, then MLH1 methylation and higher degrees of promoter methylation in general occur at a later stage of carcinogenesis.

\section{Acknowledgement}

We thank Sheryl Tripp for her assistance in performing MLH1 immunostaining.

\section{Disclosure/conflict of interest}

The authors declare no conflict of interest. 


\section{References}

1 Jass JR. Pathogenesis of colorectal cancer. Surg Clin North Am 2002;82:891-904.

2 Hawkins NJ, Ward RL. Sporadic colorectal cancers with microsatellite instability and their possible origin in hyperplastic polyps and serrated adenomas. J Natl Cancer Inst 2001;93:1307-1313.

3 Smyrk TC. Colon cancer connections. Cancer syndrome meets molecular biology meets histopathology. Am J Pathol 1994;145:1-6.

4 Samowitz WS, Albertsen H, Herrick J, et al. Evaluation of a large, population-based sample supports a CpG island methylator phenotype in colon cancer. Gastroenterology 2005;129:837-845.

5 Weisenberger DJ, Siegmund KD, Campan M, et al. CpG island methylator phenotype underlies sporadic microsatellite instability and is tightly associated with BRAF mutation in colorectal cancer. Nat Genet 2006;38:787-793.

6 Miyakura Y, Sugano K, Konishi F, et al. Extensive methylation of hMLH1 promoter region predominates in proximal colon cancer with microsatellite instability. Gastroenterology 2001;121:1300-1309.

7 Kim YH, Kakar S, Cun L, et al. Distinct CpG island methylation profiles and BRAF mutation status in serrated and adenomatous colorectal polyps. Int J Cancer 2008;123:2587-2593.

8 O’Brien MJ, Yang S, Clebanoff JL, et al. Hyperplastic (serrated) polyps of the colorectum: relationship of CpG island methylator phenotype and K-ras mutation to location and histologic subtype. Am J Surg Pathol 2004;28:423-434.

9 O’Brien MJ, Yang S, Mack C, et al. Comparison of microsatellite instability, $\mathrm{CpG}$ island methylation phenotype, BRAF and KRAS status in serrated polyps and traditional adenomas indicates separate pathways to distinct colorectal carcinoma end points. Am J Surg Pathol 2006;30:1491-1501.

10 Velho S, Moutinho C, Cirnes L, et al. BRAF, KRAS and PIK3CA mutations in colorectal serrated polyps and cancer: primary or secondary genetic events in colorectal carcinogenesis? BMC Cancer 2008;8:255.

11 Yang S, Farraye FA, Mack C, et al. BRAF and KRAS Mutations in hyperplastic polyps and serrated adenomas of the colorectum: relationship to histology and CpG island methylation status. Am J Surg Pathol 2004;28:1452-1459.

12 Sheridan TB, Fenton H, Lewin MR, et al. Sessile serrated adenomas with low- and high-grade dysplasia and early carcinomas: an immunohistochemical study of serrated lesions 'caught in the act'. Am J Clin Pathol 2006;126:564-571.

13 Eads CA, Danenberg KD, Kawakami K, et al. MethyLight: a high-throughput assay to measure DNA methylation. Nucleic Acids Res 2000;28:E32.

14 Eads CA, Lord RV, Wickramasinghe K, et al. Epigenetic patterns in the progression of esophageal adenocarcinoma. Cancer Res 2001;61:3410-3418.
15 Ogino S, Kawasaki T, Brahmandam M, et al. Precision and performance characteristics of bisulfite conversion and real-time PCR (MethyLight) for quantitative DNA methylation analysis. J Mol Diagn 2006;8:209-217.

16 Widschwendter M, Siegmund KD, Muller HM, et al. Association of breast cancer DNA methylation profiles with hormone receptor status and response to tamoxifen. Cancer Res 2004;64:3807-3813.

17 Ogino S, Nosho K, Kirkner GJ, et al. CpG island methylator phenotype, microsatellite instability, BRAF mutation and clinical outcome in colon cancer. Gut 2009;58:90-96.

18 Torlakovic E, Skovlund E, Snover DC, et al. Morphologic reappraisal of serrated colorectal polyps. Am J Surg Pathol 2003;27:65-81.

19 Ferrandez A, Pho L, Solomon C, et al. An evidencebased, multidisciplinary approach to the clinical considerations, management, and surveillance of adrenal lesions in familial adenomatous polyposis: report of three cases. Dis Colon Rectum 2006;49: 1781-1790.

20 Spirio LN, Samowitz W, Robertson J, et al. Alleles of APC modulate the frequency and classes of mutations that lead to colon polyps. Nat Genet 1998;20:385-388.

21 Tripp SR, Gedge FEH, Lyon E, et al. Microsatellite instability testing by immunohistochemistry: initial evaluation of hereditary nonpolyposis colon cancer and potential prognostic and therapeutic information. J Histotechnol 2004;27:169-172.

22 Herman JG, Graff JR, Myohanen S, et al. Methylationspecific PCR: a novel PCR assay for methylation status of CpG islands. Proc Natl Acad Sci USA 1996;93:98219826.

23 Ogino S, Cantor M, Kawasaki T, et al. CpG island methylator phenotype (CIMP) of colorectal cancer is best characterised by quantitative DNA methylation analysis and prospective cohort studies. Gut 2006;55:1000-1006.

24 McGivern A, Wynter CV, Whitehall VL, et al. Promoter hypermethylation frequency and BRAF mutations distinguish hereditary non-polyposis colon cancer from sporadic MSI-H colon cancer. Fam Cancer 2004;3:101-107.

25 Halvarsson B, Lindblom A, Johansson L, et al. Loss of mismatch repair protein immunostaining in colorectal adenomas from patients with hereditary nonpolyposis colorectal cancer. Mod Pathol 2005;18:1095-1101.

26 Rijcken FE, Hollema H, Kleibeuker JH. Proximal adenomas in hereditary non-polyposis colorectal cancer are prone to rapid malignant transformation. Gut 2002;50:382-386.

27 Farris AB, Misdraji J, Srivastava A, et al. Sessile serrated adenoma: challenging discrimination from other serrated colonic polyps. Am J Surg Pathol 2008; 32:30-35.

28 Goldstein NS. Serrated pathway and APC (conventional)-type colorectal polyps: molecular-morphologic correlations, genetic pathways, and implications for classification. Am J Clin Pathol 2006;125:146-153.

Supplementary Information accompanies the paper on Modern Pathology website (http://www.nature.com/ modpathol) 